\title{
Adopting the Principle of Closed Set to People Identification Based on Human Hand Geometry
}

Maha Abd Alrahman Al-Hasoo, Zeena N. Al-kateeb

Department of Computer Sciences, College of Computer Sciences and Mathematics,

University of Mosul, Mosul, Iraq

Received on:24/10/2010

E-mail address: zeenaalkateeb@yahoo.com

\begin{abstract}
We propose a Identification System Based on Human Hand Geometry Features, we extract 50 features to use it in recognition process. By constructing the principle of Close Set Identification System, the system database has been built using modified digital scanner. The system were tested on database contain 500 images referred to 50 persons, 10 images for each person.

The evaluation of the system performance was calculated by using three matching metrics that are Absolute Distance, Euclidean Distance, D1 Distance and get on the highest recognition rate using Absolute Distance more than 97\%.

MATLAB 7.9.0(2009b) programming language has been used to execute the paper algorithms, because its facilities in processing digital images.

Keyword: Identification, Hand Geometry, close Set

$$
\begin{aligned}
& \text { تبّني مبدأ عمل المجموعات المغلقة لتمييز الأثخاص اعتماداً على الأبعاد الهندية لليد البشرية }
\end{aligned}
$$

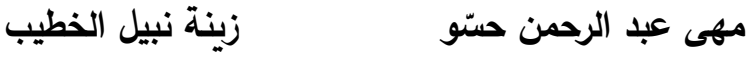

$$
\begin{aligned}
& \text { كلية عوم الحاسبات والرياضيات, جامعة الدوصل لبن }
\end{aligned}
$$

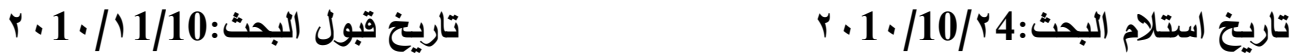

\section{الملخص}

Hand تم اقتراح نظام للتعرف على الاشخاص بالاعتماد على خصائص الابعاد الهنسية لليد البشرية Geometry ، اذ تم استخلاص 50 خاصية من تلك الخصائص بهدف استخدامها لعملية التمييز • وبتبني مبدأ عمل انظمة التعرف على المجموعات المغلقة Close Set Identification System ـ تم بناء قاعدة بيانات النظام باستخدام جهاز الماسح الضوئي الذي تم تحويره لهذا الغرض وتجربة النظام المقترح على قاعدة بيانات تضم

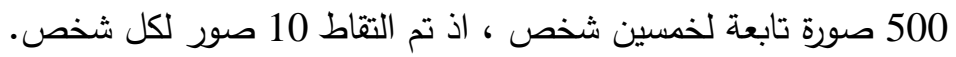

تم قياس مدى كفاءة النظام باستخدام ثلاث مقاييس من مقاييس التطابق matching metric المسافة المطلقة Absolute Distance و المسافة الاقليدية Euclidean Distance والمسافة الدالية الاولى D1 Distance وقد تم الحصول على اعلى نسبة تمييز باستخدام مقياس المسافة المطلقة Absolute Distance والتي تجاوزت 97\% تم استخدام لغة (2009b) لتنفيذ خوارزميات البحث والتي توفر تسهيلات التعامل مع 
أخذت أنظمة الوثوقية القائمة على استخدام المقاييس الحيوية بالانتشار بشكل كبير مقارنة بأنظمة الوثوقية

التقليدية وذلك لدقتها وقدرتها على التمييز بسهولة بين الثخصية الحقيقية للمستخدم ومنتحلي الثخصية لاعتمادها على سمات شخصية في التعرف على المستخدم ، بالإضافة إلى أن هذه الأنظمة تلقى قبولاً كبيرًا بين المستخدمين

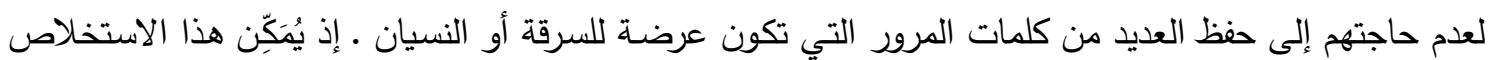
للخصائص الفردية من التعامل مع الأشخاص بسرعة وبدقة عالية ـ وقد حددت عدد من الخصائص التي سميت

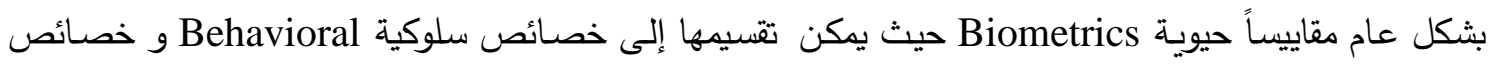
جسدية Physiological . تمثل الخصـائص السلوكية أنماطا سلوكية متوافقة تميز كل فرد. أمـا الخصـائص الجسدية فتشير إلى نمط هيكلي أو شكلي يتضح من خلال جسد الإنسان [2].

2- طرق الوثوقية Authentication Methods

تعرف الوثوقية Authentication على أنها منح الثخص الصحيح التخويلات والصـلاحيات الملائمسة

$$
\text { وإمكانية الوصول الصحيح في الوقت الملائم [17] ـ }
$$

يمكن تصنيف مشكلة وثوقية الثخص Personal Authentication إلى صنفين أساسيين حسب التعقيد وكما

هو موضح بالثكل (1) (1) من من

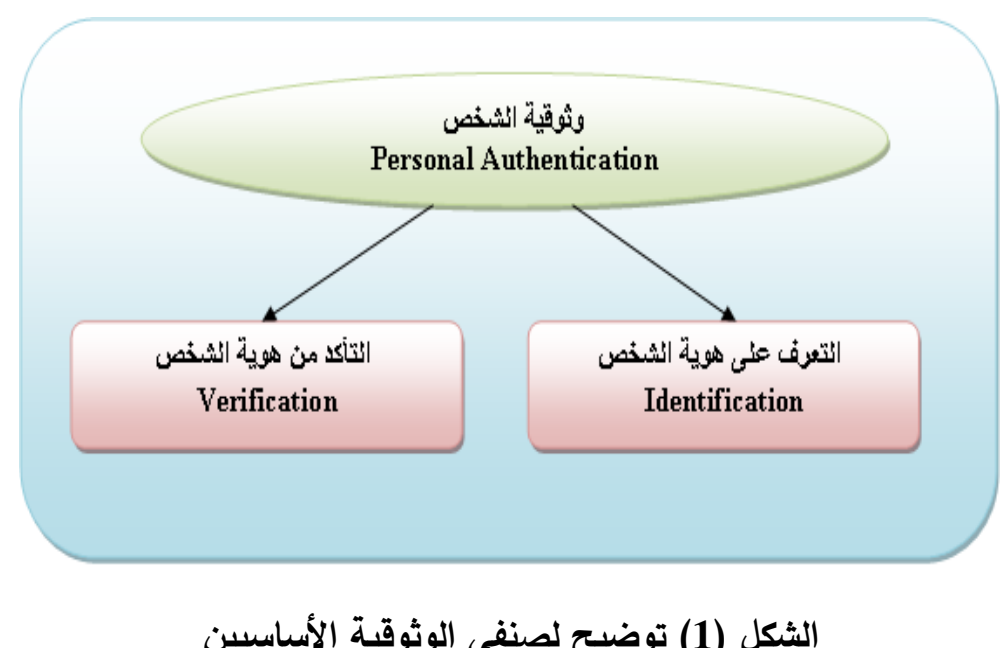

أ- مشكلة التعرف على هوية الثخص Identification Problem

يتم التعرف على هوية الثخص المدخلة بياناته من بين مجموع الأشخاص المسجلين ضمن قاعدة بيانات

النظام [13].

يمكن وصف مشكلة التعرف على هويـة الثخص على أنها واحد ـ. إلى . العديد (one_to_many) ، حيث تتم

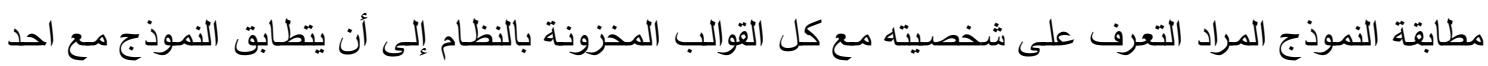

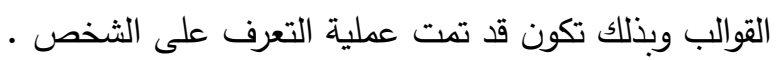
يمكن تقسيم أنظمة التعرف على الأثخاص الى العديد من الانواع تبعا لطريقة التعامل معها منها : 
1. أنظمة التعرف المغلقة Closed Set Identification : حيث يكون من المعلوم في مثل هذه الأنظمة أن الثخص موجود ضمن قاعدة البيانات . 2. أنظمة التعرف المفتوحة Open Set Identification : وفيها يكون من غير المؤكد وجود بيانات الثخص

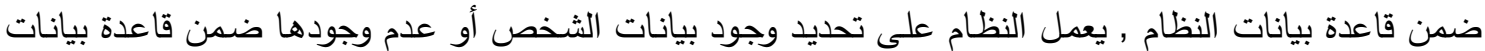
النظام[15].

ب _ مشكلة التأكد من هوية الثخص Verification Problem يتم تحديد كون الثخص المعني هو الثخص الحقيقي ( الثخص الهدفCClaimed Identity ) أم هو مجرد منتحل للشخصية[13]. يمكن وصف مثكلة التأكد من هوية الشخص على انها واحد ـ الى . واحد (one_to_one) ) ، حيث تتم مطابقة

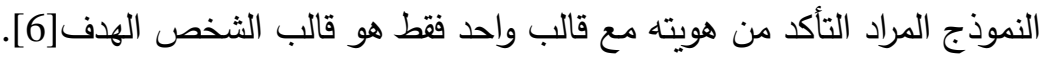

3-استخدام الابعاد الهنذية لحل مثكلة الوثوقية

تعد الابعاد الهندسية لليد البشرية من المقاييس الحيويـة الفسيولوجية المستعملة على نحو واسـع في العي المجالات التجارية منذ أكثر من 20 عام , حيث شهذ العام 1985 ظهور أولى الأنظمة المستخدمة للتحقق من التهن وثوقية الثخص والقائمة على أساس هنسية اليد تلى ذلك وبعد فترة وجيزة توفر هذه الأنظمة على المستوى التجاري

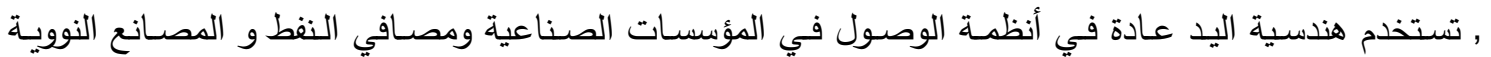

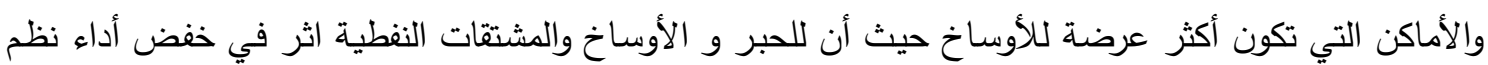

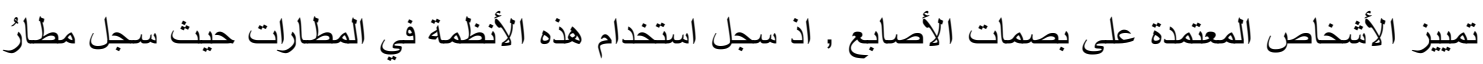
سان فرانسيسكو الدولي استخدام أنظمة هندسية اليد للسيطرة على وصول الدستخدم منذ عام 1993 [11].

\section{Hand Geometry of Hunan Hand الابعاد الهنسية لليد البشرية}

تضم اليد البشرية العديد من انواع الكقاييس الحيوية حيث انها تضم بصمة الاصابع Fingerprint بصمة الكف Palm print , بصمة الظفر اليد Hand geometry تستتد تقنية الابعاد الهنسية لليد Hand geometry على حقيقة كون كل شخص تقريبا يمتلك شكل يد مميز له , لا يتأثر كثيرا بعوامل الزمن والثيخوخة حيث يكون حجم اليد يكون ثابتا نسبيا اذا كان الانسان قد اكمل

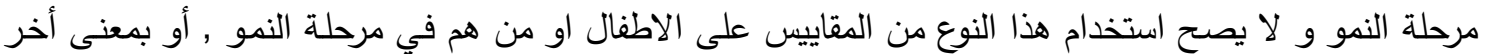

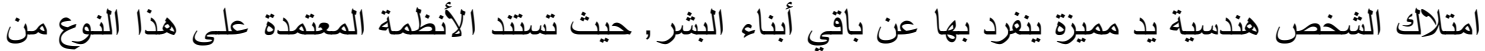

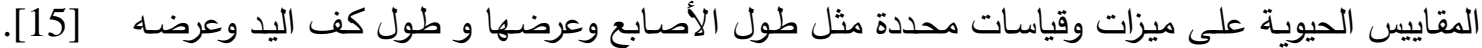


هناك عدة محاسن وميزات لاستخدام تتنية الابعاد الهندية لليد البشرية Hand geometry في مجال

التوثق من الشخصية Personal Authentication نوجز منها:

سهولة الاستخدام Ease Of Use: [11]

[11]: Resistant to spoofing مقاومة الغش

[11] : Small template size صغر حجم النماذج

[4] High Ability Of Readers قدرة أجزة قراءة مقاييس اليد الهندسية العالية

[11] Reliability of Technique وثوقية التقنية

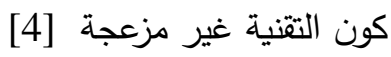

[8] Low Resolution Images الدقة المنخفضة للصور Non-Intrusive

[9] Fast results سرعة الحصول على النتائج

سهولة التتظيف والإصلاح بالنسبة للاجهزة المستخدمة في هذه التقنية [15].

6isadvantages Of Hand Geometry Technique مساوئ تقنية هندسية اليد

وجوب التواجد الجسدي (مع تطور الشبكات تم معالجة هذه المشكلة) .

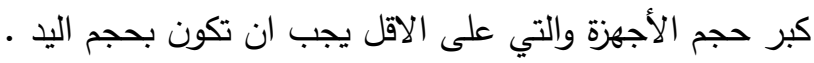

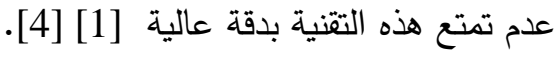

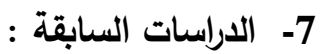

ومن أشهر من عمل في هذا مجال التمييز على أساس الأبعاد الهندسية لليد البشرية,Jain لوآخرون [7] إذ

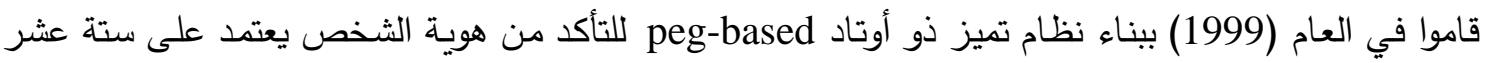

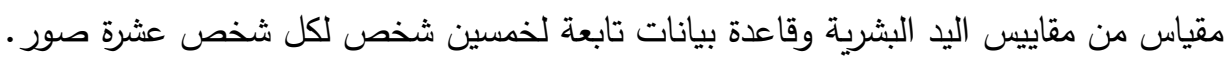

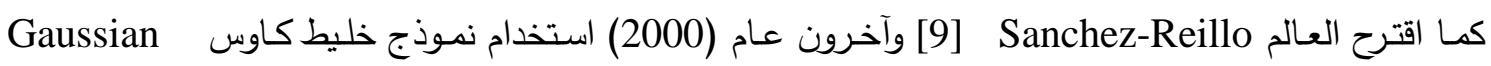
mixture model (GMM)

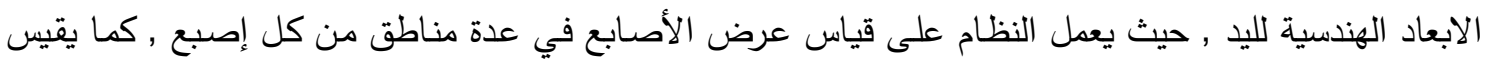

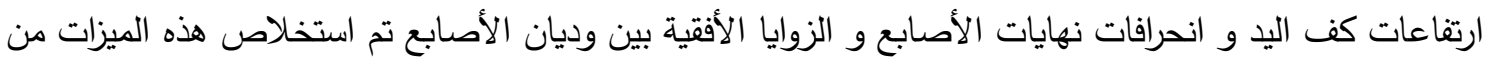

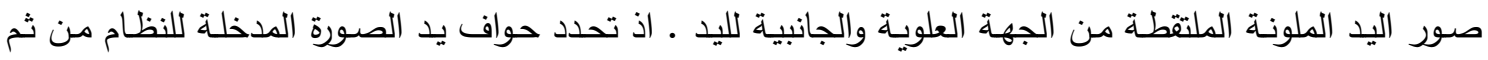
تجرى عليها عمليـات التحليل المورفولـوجي morphological analysis ليتت بعدها الحصـول على متجـهـ - Feature Vector الخصائص

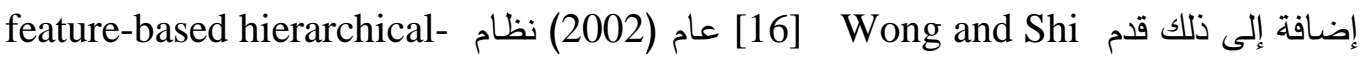

Finger Widths والمعتمد على طول الأصـابع -framework for hand geometry recognition وعرضها Finger Lengths , والخطوط الأساس مابين الأصـابع Interfinger Baselines , كما انه اعتمد

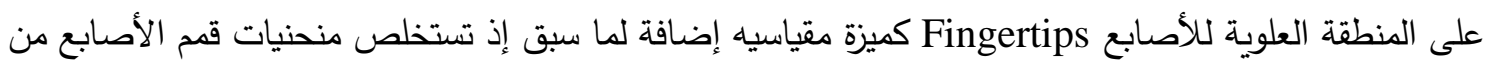

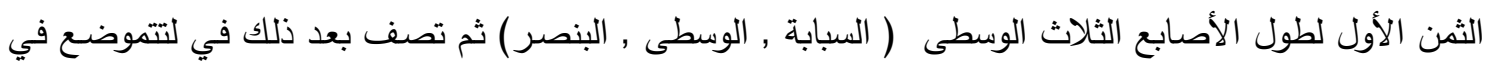

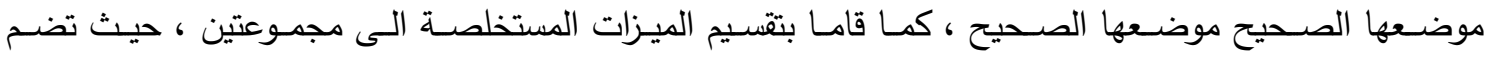


المجموعـة الأولى 13 ميزة فيما تضم المجموعة الثانية 3 ميزات فقط يلي ذلك استخدام نموذج خليط كاوزن

Euclidean distance لتميز خصائص المجموعة الأولى و المسافة التقليدية Gaussian mixture model لإجراء عملية المقارنة على خصائص المجموعة الثانية ـ المانير

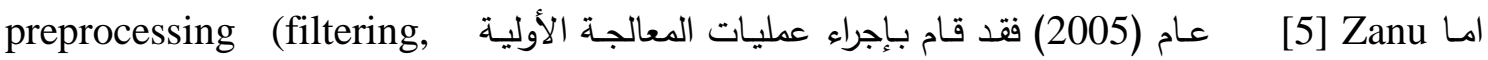
على الصور قبل استخلاص الsقاييس الهندسية منها ، حيث كان Binarization and contour detection) يقوم باستخلاص تسعة خصائص Features من أربعة أصابع (الخنصر و البنصر و الوسطى و السبابة ) وهي

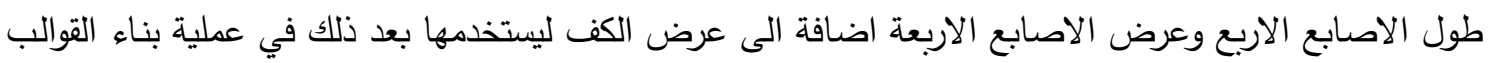
المرجعية وإجراء عمليات المقارنة ,اعتمد Zanu على الثبكات العصبية في عمليتي استخلاص الخواص واجراء Multilayer Perceptron Networks عملية المقارنة حيث قام باستخدام شبكة البرسبترون متعددة الطبقات علئات • لهذا الغرض قدم Sharma واخرون [3] في (2008) نظاما مبتكرا للتأكد من هوية الثخص بالاعتماد على هندسية اليد ثلاثية الأبعاد ,حيث قام النظام باستخدام projected light patterns لاستخلاص خصائص البعد الثالث لكف اليد (العمق depth map of the palm ) بدلا من حساب هذه الخصائص بالطرق التقليدية المعروفة , يتت

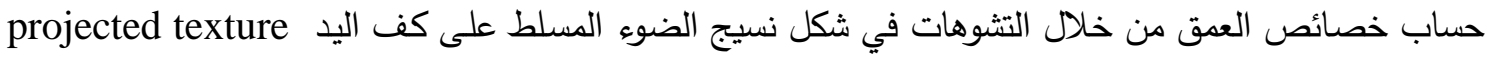
pattern

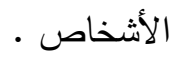

\section{Hand Geometry Recognition System النظام المقترح لتمييز هندسية اليد}

بصورة عامـة يمر نظـام التمييز القائم على أسـاس الابعاد الهندسية لليد بالمراحل الأولية الثلاثتة وهي

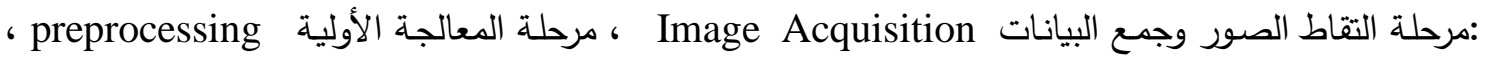
مرحلة استخلاص الميزات Features Extraction وبانتهاء هذه المرحلـة نكون قد حصلنا على الميزات والخصائص Features and characteristics التي سيستخدمها النظام مستقبلا في عملية التمييز •

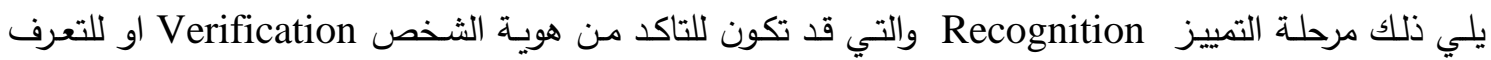
على الثخص Identification والثكل (3) يوضتح المراحل التي يمر بها اي نظام تمييز قائم على اسـاس هول المقاييس الحيوية بصورة عامة . 


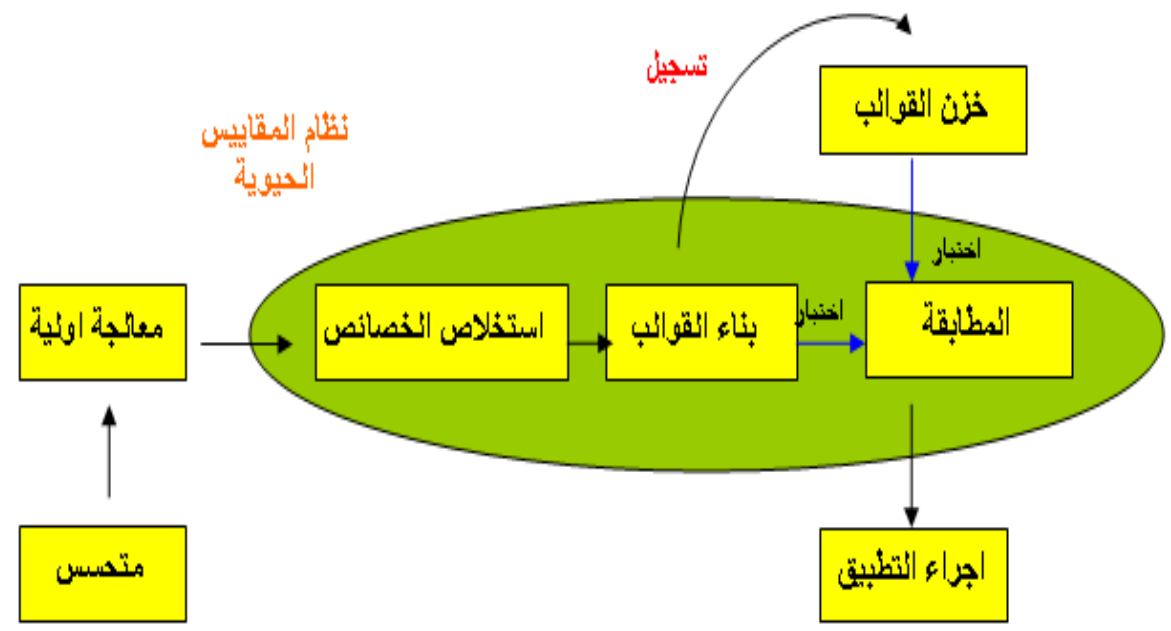

الثكل (3) يوضح المراحل التي يمر بها اي نظام تمييز قائم على اساس المقاييس الحيوية بصورة عامة .

\section{Data Collection 9-1 مرحلة التقاط الصور وجمع البيانات}

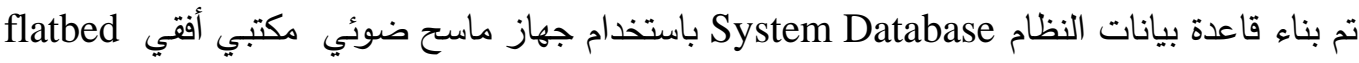
desktop document scanner

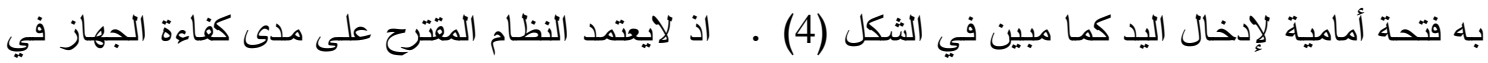

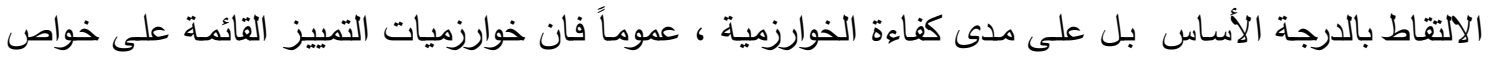

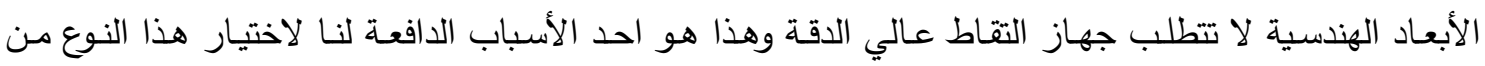
المقاييس الحيوية حيث كان الهدف من البحث هو الوصول إلى تمييز متوسط الدقة وبكلف متوسطة أو منخفضة.
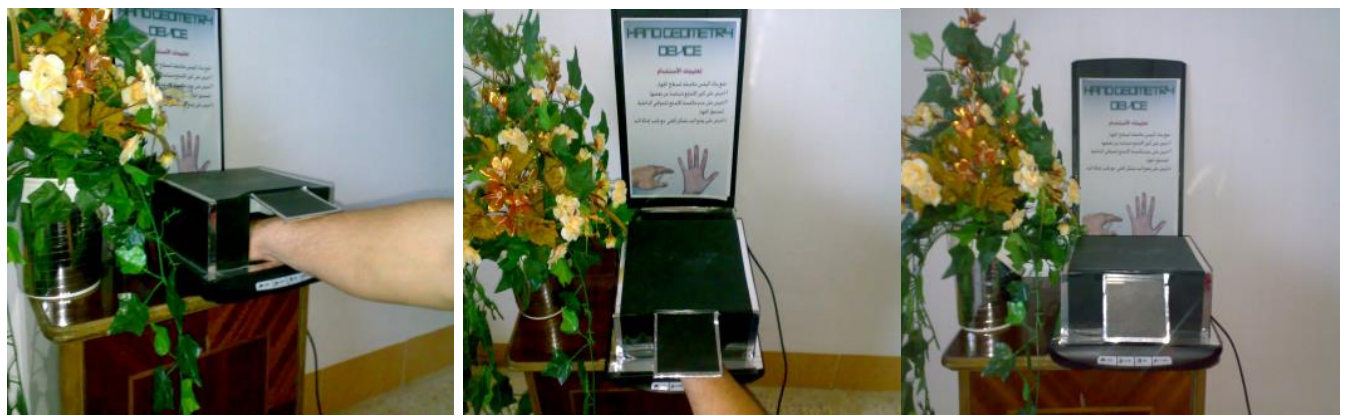

الثكل (4) يوضح شكل جهاز الماسح الضوئي المحور المستخدم لالتقاط الصور و جمع قاعدة البيانات

اذ تم التقاط صور أيدي أثخاص تتراوح أعمارهم مابين 20-50 عام ومن كلا الجنسين. ، حيث يعمل الصندوق على حجب الضوء الخارجي مما يساعد على تقليل الظل والضوضاء reduce noise and shadow ويساهم في الحصول على صور جيدة للتطبيق ، حيث يقوم المستخدم بإدخال يده اليمنى right hand من خلال فتحة الجهاز ويضعها ملاصقة لسطح جهاز الماسح الضوئي مع مراعاة عدة نقاط : 
وجوب وضع اليد ملاصقة للسطح الداخلي للجهاز •

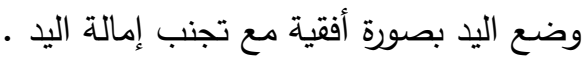

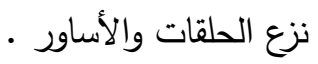

يجب التقاط 3 صور لإتمام عملية التسجيل .

يجب أن يكون الأشخاص المسجلين بالنظام قد تجاوزوا سن النمو (لا يصح تطبيق النظام على الأطفال)

يكون النظام Peg-free بدون أي أوتاد لتحديد موضع اليد ولكن يراعى وضع اليد بصورة صحيحة مما

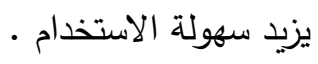

وجوب كون طول الأظافر معتدل إذ أن الأظافر الطويلة تسبب مشاكل بالنظام .

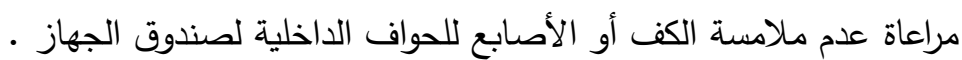

، jpg تلتقط صور الأيدي عن طريق الجهاز وتخزن كصور ملونة 1120 colored image تتطلب مرحلة التسجيل التقاط ثلاث صور ليد المستخدم , أما مرحلة التحقق من الشخصية فإنها تتطلب التقاط

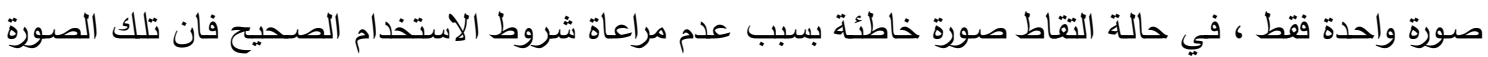

$$
\text { تهمل ويطالب المستخدم بالتقاط صورة أخرى . }
$$

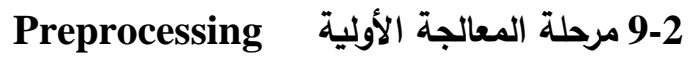

يكمن إجمال خطوات المعالجة الأولية بات

ت و colored image الى صورة ذات تدرجات رمادية grayscale image

تحويل الصورة ذات التدرجات الرمادية grayscale image إلى صورة ثنائية Binary image ، يمكن

التقاط الصورة مباشرة كصورة رمادية كما يمكن تحويل الصورة من صورة ملونة الى صورة ثنائية مباشرة كما الا ان التحويل من صورة ملونة الى صورة ذات تدرجات رماديـة ثم الى صورة ثنائية يعطي نتائج افضل ، وكما مبين

بالشكل (5).

• تطبيق مرشحات التحسين Enhancement Filters لإزالة الضوضساء و تقليل التعرجات في حواف اليد حيث تم استخدام مرشح المعدل Average اولا ثم استخدام مرشح القرص Disk يليه استخدام مرشح تلقليل

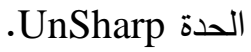

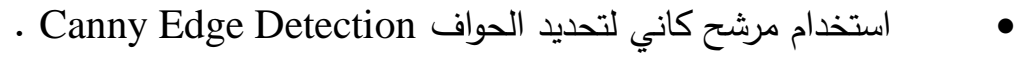
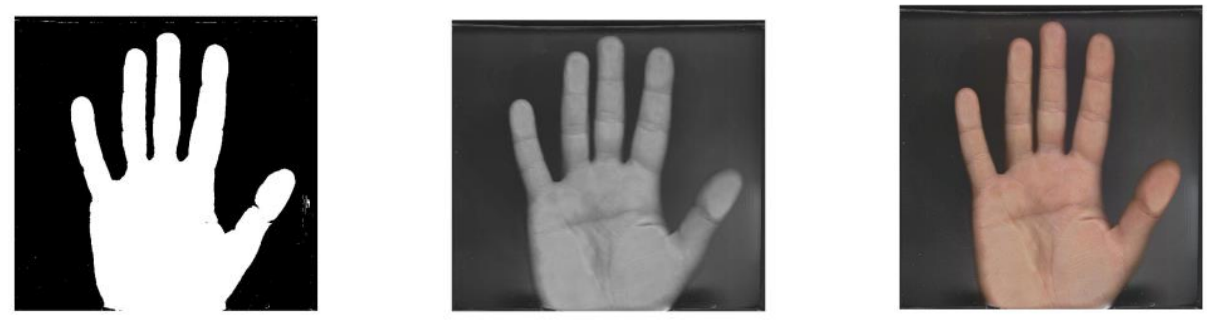

الثكل (5) يوضح الصورة الاصلية ونتائج تحويلها الى صورة رمادية اولا ثم الى صورة ثنائية

Feature Extraction 9-3 مرحلة استخلاص الخواص 
في نهاية مرحلة المعالجة الأولية preprocessing استطعنا الحصول على صورة سوداء تحتوي على مجموعة من النقاط البيضاء التي تصف كل الحواف الموجودة في الصورة الأصلية ، حيث تعد الحافة الأطول والأبرز في تلك الصورة هي الحافة التي تمثل حدود شكل اليد Boundary of Hand Shape ، يتم بعد ذلك

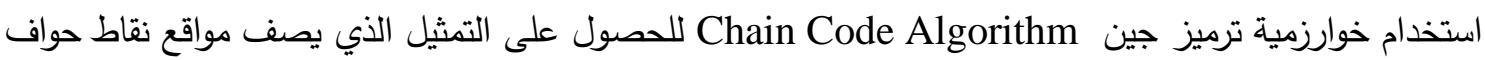

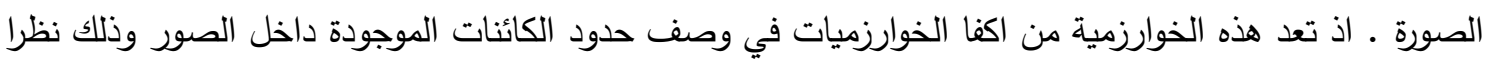

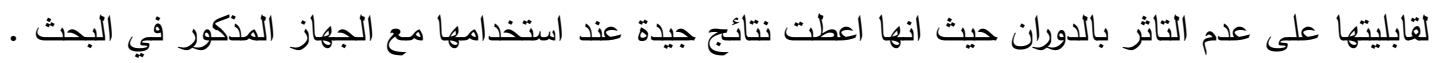
تلي هذه الخطوة خطوة إيجاد النقاط المرجعية landmarks حيث نقوم باستخلاص تسعة نقاط مرجعية

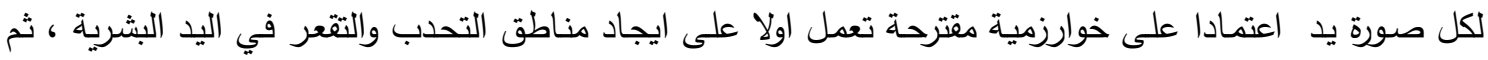
تحديد النقطة الوسطيه من نقاط هذه المناطق على انها النقطة المرجعية التابعة لتلك المنطقة .

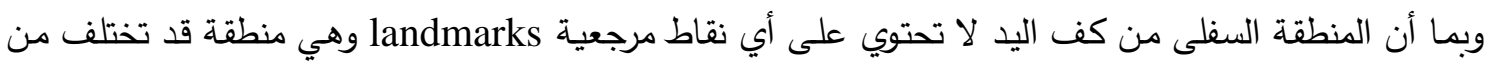
شخص لأخر إضافة لإمكانية اختلافها بالنسبة للشخص الواحد ، إذ تختلف حسب مقدار إدخال اليد في الجهاز لذا لذا نعمل على إهمال الجزء السفلي من صورة اليد وذلك لتقليل عدد نقاط حواف اليد مما يزيد سرعة المعالجة ، والثكل

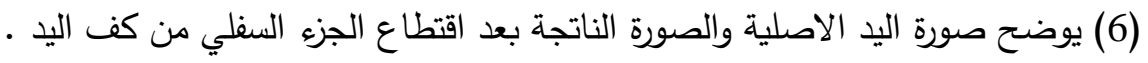
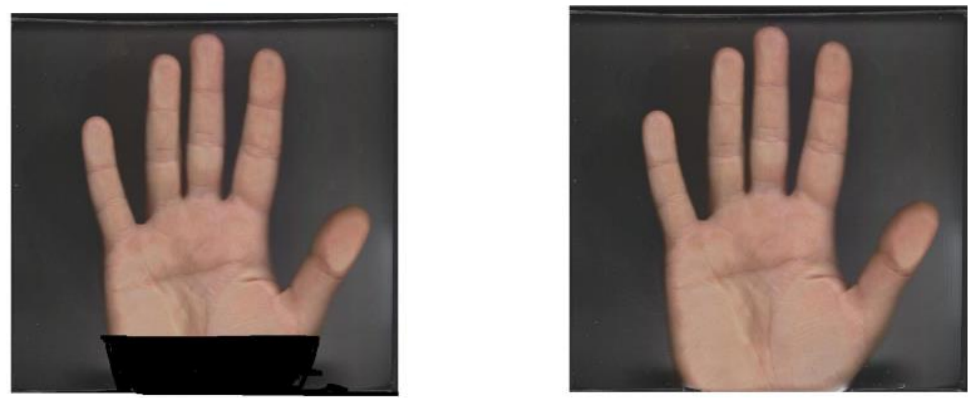

الثكل (6) يوضح الصورة الاصلية والصورة المقتطع منها الجزء السفلي من كف اليد

يجب أن يكون عدد النقاط المرجعية landmarks المستخلصة من صورة يد المستخدم السليم (الذي يملك

$$
\text { خمسة أصابع ) تسعة نقاط وهي : نحون عدون }
$$

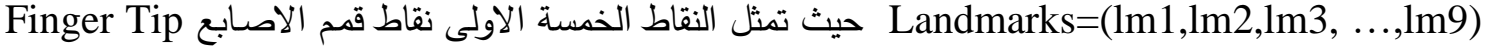
في حين تمثل النقاط الاربع التالية نقاط الوديان المحصورة بين الاصـابع Finger Valley ، في حالة كون عدد النقاط المرجعية اقل او اكثر من تسعة نقاط دل ذلك على وجود خطا في وضع اليد ، عندها يتوقف النظام عند

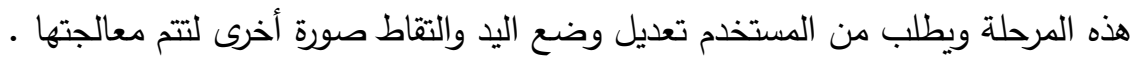
هناك عدد كبير من الخصائص التي من الممكن استخلاصها من شكل اليد و الأصسابع ، إذ يوجد ما يقارب 90 خاصية من خصائص اليد الهندية ، قمنا في نظامنا المقترح باستخلاص 50 خاصية منها واستخدامها لهدف

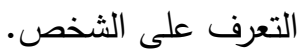

Finger Base Lines 9-3-1 
تعرف خطوط الأصـابع الأساسية Finger Base Lines على أنها تلك الخطوط الواصلة بين نقطتي

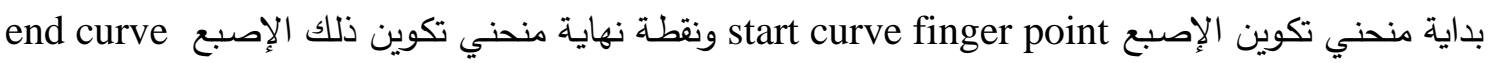

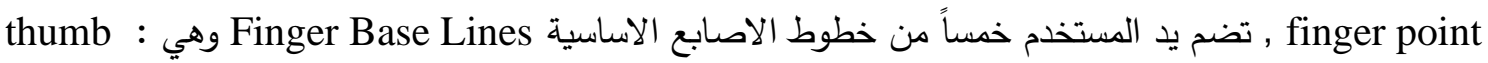
(baseline , index baseline , middle baseline , ring baseline ,little baseline )

\section{Finger Lengths أطوال الأصابع 9-3-2}

تعد من أشهر الخصائص وأكثرها استخداماً في أنظمة التمييز القائمة على أساس خصائص اليد الهندية

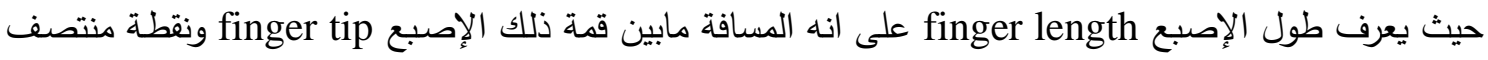

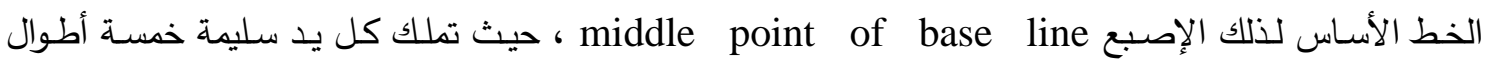
(L1,L2,L3,L4,L5)

\section{Finger Widths 9-3-3}

يختلف الناس عن بعضهم البعض بعرض الأصابع فهناك من يملك يد بأصـابع عريضـة ومنهم من تمتاز أصابعهم بالنحافة ومنهم مابين هذا وذاك ، كما أن عرض إصبع الثخص نفسه قد يختلف من منطقة لأخرى حيث

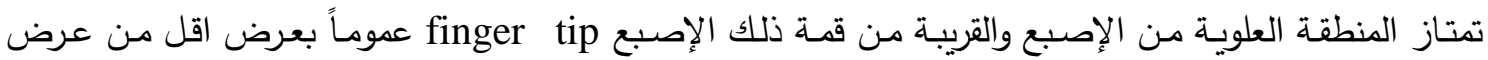

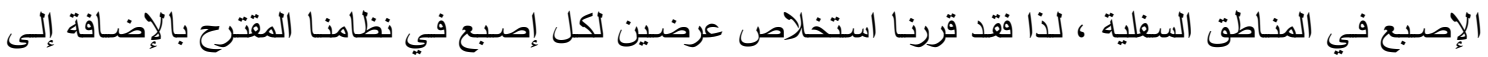
الخطوط الأساس للأصابع base lines إذ أنها تعتبر بمثابة العرض الثالث لكل إصبع، وبذلك يكون لدينا ثلاث مقاييس لعرض كل إصبع أي خمسة عشر مقياس للأصابع الخمسة . $\mathrm{FW}=(\mathrm{W} 1.1, \mathrm{~W} 1.2, \mathrm{~W} 1.3, \mathrm{~W} 2.1, \mathrm{~W} 2.2, \mathrm{~W} 2.3$ .W5.1,W5.2,W5.3)

Palm Width 9-3-4 عرض الكف

أثناء جمع البيانات تم ملاحظة اختلاف الأفراد في شكل كف اليد palm shape ، حيث يملك البعض

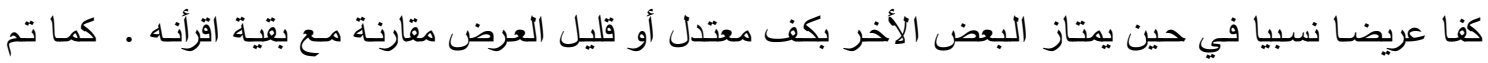

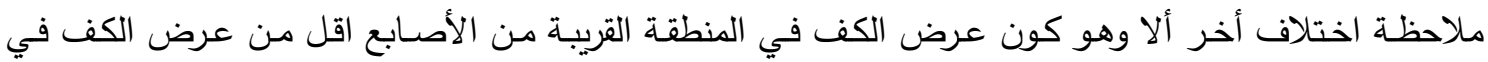

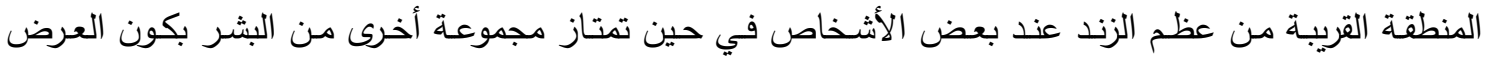

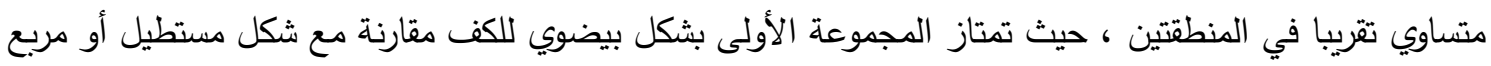
لشكل اليد بالنسبة للمجموعة الثانية Palm Width=(PW1,PW2)

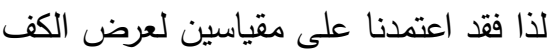
يقيس الأول عرض الكف في المنطقة الموازية لبداية منحني تكوين إصبع الإبهام first width of palm ويقيس الثاني عرض الكف في المنطقة الموازية لنهاية منحني تكوين إصبع الإبهام second width of palm

Palm Length ول بول الكف

كما أن البشر مختلفون في عرض الكف palm width فإنهم مختلفون أيضا في طول الكف ، حيث تم الاعتماد على هذه الميزة كإحدى الخصائص التميزية في نظامنا المقترح ، يعرف طول الكف palm length على 
انه المسافة مابين نقطة قمة الإصبع الأوسط middle finger tip point ونقطة منتصف العرض الثاني للكف palm width $=(\mathrm{pl})$ ، middle point of second palm width

\section{Finger Top Regions 9-3-6}

تعد مساحة المنطقة العلوية لقمم الأصـابع من الخصائص المميزة للأفراد ، اذ تثكل منطقة قمة الإصبع

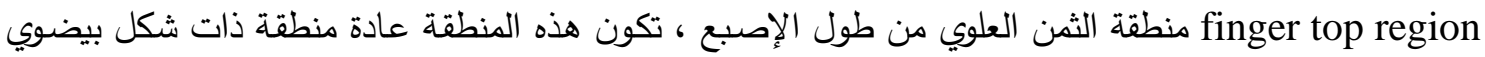
لذا تحسب مساحتها اعتمادا على القانون التالي : مساحة الثكل البيضوي =نق المحور الكبير × نق المحور الكبير × النسبة الثابتة حيث اعتمدت مساحة قمة الأصـابع كجزء من الخصائص التميزيـة في نظامنا ، بالإضـافة لكون مساحة المنطقة العلوية لقمم الأصابع من الخصائص المميزة للأفراد فقد لاحظنا اختلاف الأفراد في مقدار تحدب الأصابع ، فهنالك أفراد ذوي تحدب أصابع عالي وهنالك أفراد آخرين ذوي تحدب أصابع واطئ ومنهم من هو بين هذا وذاكي ، ونظرا

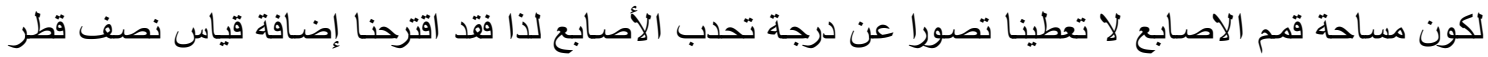
المحور الكبير max_radias_of_finger_radias_of_finger وقياس نصف قطر المحور الصغير الخصائص التميزية للنظام ، إذ أنها تعطينا تصوراً واضحاً عن مدى تحدب الأصابع وكما مبين في الثكل (8).

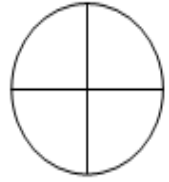

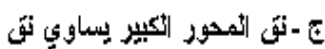
| أمحور التصنير العبير

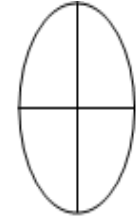

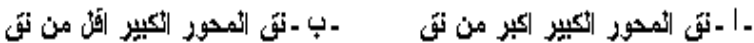

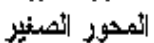

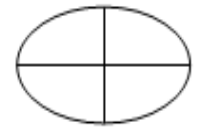
|نمحور انصينير

الشكل(8) يوضح أشكال قم الأصابع عند مختلف البشر

\section{Line Segments and Angle Values}

9-3-7 المقاطع الخطية وقياس الزوايا المحصورة بينها

تعرف بعض المقاطع الخطية Line Segments الواصلة بين بعض النقاط المرجعية Landmarks

وقياس الزوايا المحصورة بين تلك المقاطع الخطية Angle Values على أنها من الخصائص الهندية المميزة لليد البشرية ، حيث انه من الممكن استخدامها في أنظمة التمييز القائمة على أساس الخصائص الهندسية لليد البشرية. أضيفت إلى الخصائص التي يقوم نظامنا المقترح بالاعتماد عليها بهدف تمييز الأفراد والثكل (9) يوضـح المقاطع الخطية والزوايا المحصورة بينها التي أُعُمِدِت كخصائص تميزية في نظامنا المقترح 

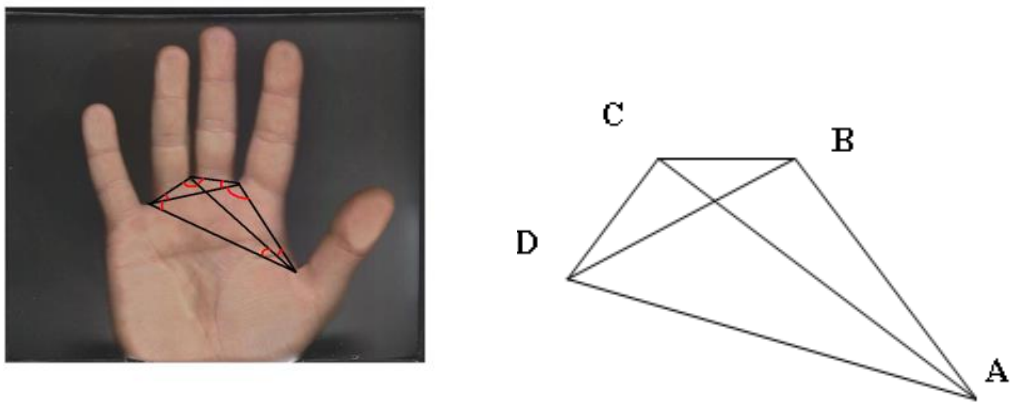

الثكل (9) يوضح المقاطع الخطية والزوايا المحصورة بينها التي أُعتُمِدت كخصائص تميزية في نظامنا المقترح

Line Segments 9-3-8

المقطع الخطي هو الخط الواصل مابين نقطة وادي الى نقطة وادي اخر تم اختيار اربعة مقاطع خطية وهـي (DA و CA و BA و DB انظر الثـكل (9) ، ونظـرا لاعتمـاد إيجـاد الخطـوط المـكورة على النقـاط المرجعية landmarks والتي تكون عادة ثابتة بالنسبة للشخص ذاته ، فانه من المككن إيجاد الخطوط ذاتها عند التقاط أكثر من صورة للشخص الواحد مع ملاحظة إمكانية وجود اختلاف طفيف عند التقاط أكثر صورة للشخص ذاته بسبب الاختلاف في وضع اليد ولكن هذا لا يخرجنا من إطار اعتبارها خاصية تميزيه

\section{Angles between Line Segments}

9-3-9 الزوايا المحصورة بين المقاطع الخطية وتمثل الزوايا المحصورة بين المقاطع الخطية وقد تم ايجاد 8 زوايا وهي (BAC و CAD و BDA و

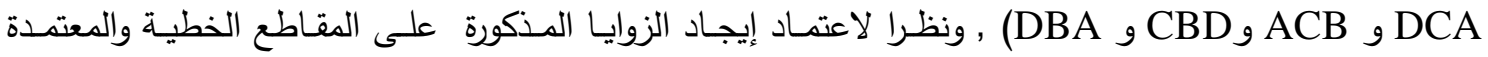
أساسا على النقاط المرجعية التي تعد نقاطا ثابتة بالنسبة للشخص الواحد فانه من المككن ايجاد نفس الزوايا عند التقاط اكثر من صورة ليد المستخدم • يتم حساب قياس الزوايا المذكورة اعتمادا على قانون الجيوب فاذا أردنا حساب قياس الزاوية CBD مثلا فان ذلك يتم اعتمادا على القانون التالي : $\angle C B D=\arccos \left(\left((B C)^{2}+(B D)^{2}-(C D)^{2} / 2(B C)(B D)\right)\right.$ و الثكل (10) يبين الصسورة الاصلية والصـورة الناتجـة من مرحلة استخلاص النتائج كمـا ان الجدول (1) يبين

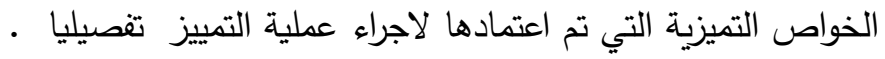
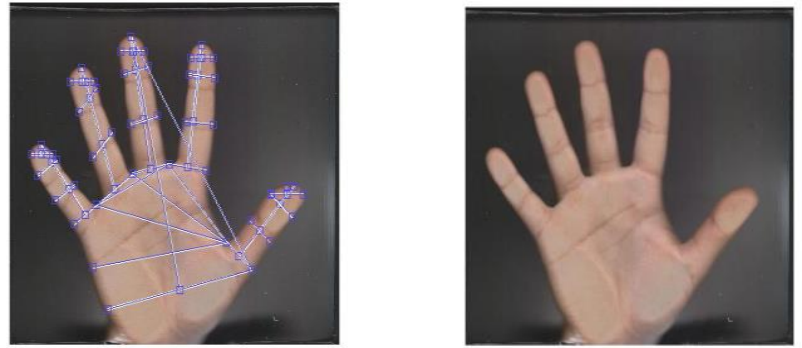

الشكل (10) يوضح الصورة الاصلية والصورة الناتجة من مرحلة استخلاص النتائج 
الجدول (1) يبين الخواص التميزية التي تم اعتمادها لاجراء عملية التمييز

\begin{tabular}{|c|c|}
\hline أثعدد & أخاصبة \\
\hline 5 & 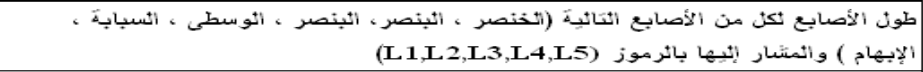 \\
\hline 5 & 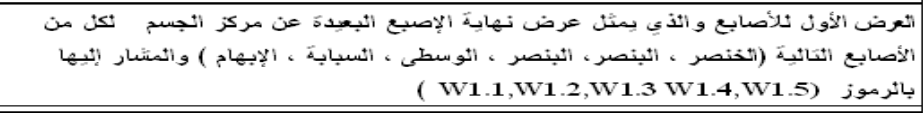 \\
\hline 5 & 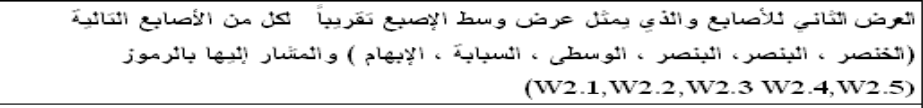 \\
\hline 5 & 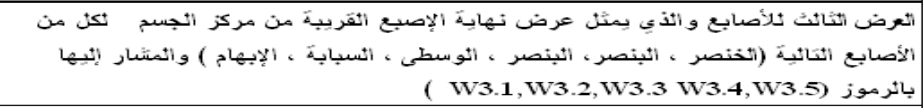 \\
\hline 2 & 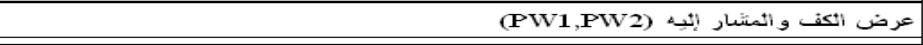 \\
\hline 1 & طون ألكف و المششار إلثيع (p1) \\
\hline 5 & 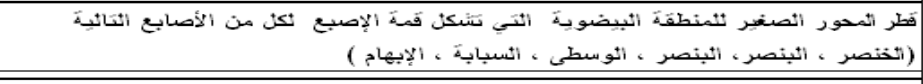 \\
\hline 5 & 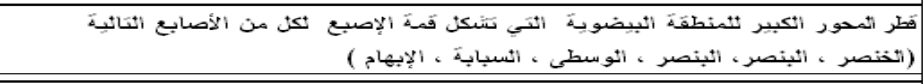 \\
\hline 5 & 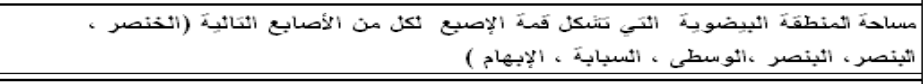 \\
\hline 4 & المقاطع الخطبة و أثمشار إلثيها (DB ، BA ، CA ، DA) \\
\hline 8 & 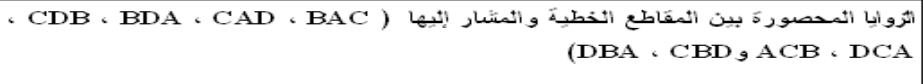 \\
\hline 50 & مجموع الخو أص أكثي \\
\hline
\end{tabular}

Enrollment 9-4 مرحلة التسجيل

يتطلب بنـاء أي نظام تمييز قائم على أسـاس الخصـائص الهندسية لليد البشرية تكوين قاعدة بيانـات تضم جميع القوالب المرجعية Reference Template التابعة للأشخاص المسجلين بالنظام ، لذا تعد لتصني عملية بناء القوالب المرجعية Reference Template من اهم الخطوات التي يمر بها النظام حيث تتم هذه الخطوة في طور التسـيل ، اذ يتطلب النظام المقترح التقاط ثـلاث صـور ليد المستخدم يلي ذلك استخلاص الخصائص التميزية من كل صورة من الصور الثلاثة ثم يبنى القالب التابع للشخص المحدد من خلال إيجاد معدل

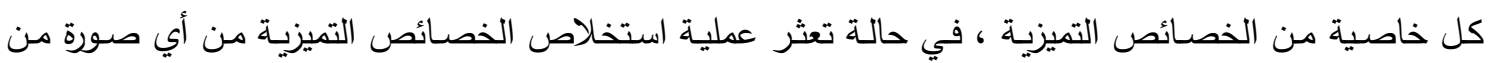
الصور الثلاثة فان النظام يطلب من المستخدم التقاط صورة بديلة لإتمام عملية التسجيل ، حيث قمنا باجراء هذه

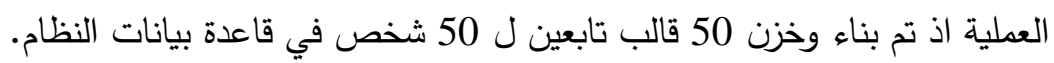

Matching or Classification 9-5 مرحلة المطابقة أو التصنيف

accept حيث يتم الحكم اما بقبول الثخص هذه المرحلة مرحلة اتخاذ القرار make decision تعد على انه شخص مخول ومسجل بالنظام ،او برفض الثخص reject user وتعريفه على انه مجرد متطفل ومنتحل للشخصية ، وبما ان النظام مبني لغرض التعرف على الشخصidentification the user وتميزه من بين

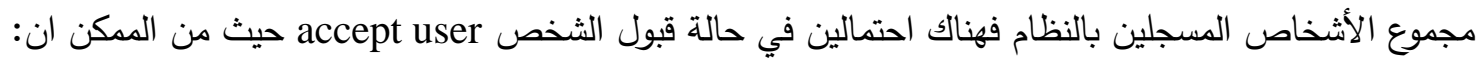
يعرف الثخص على انه شخص مخول ومسجل بالنظام وتميز شخصيته بصورة صحيحة يعرف الثخص على انه شخص مخول ومسجل بالنظام وتميز شخصيته بصورة خاطئة 
وفي نظامنا المقترح فقد تم استخدام ثلاث مقاييس من مقاييس التطابق matching metric

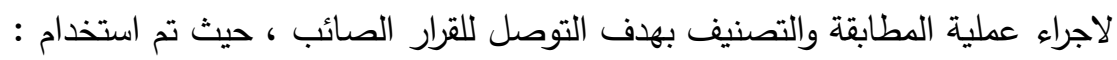
Absolute Distance المسافة المطلقة

$$
\begin{aligned}
& D_{a}=\sum_{i=1}^{d}\left|y_{i}-f_{i}\right| \\
& \text { والتي تعتمد على القانون التالي : }
\end{aligned}
$$

\section{Euclidean Distance المسافة الاقليدية}

بالاعتمـاد على قاعدة بيانات النظام والمكونـة من 500 صورة تابعة لـ 50 شخص حيث تضم قاعدة

البيانات 10 صورملونة لكل شخص ، وباستخدام 50 خاصية تميزية مستخلصة من الصور الملتقطة.

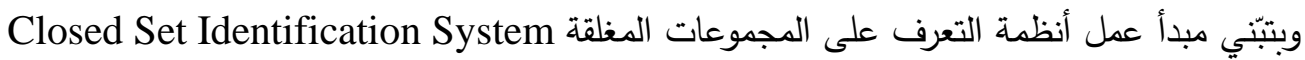
تم التمييز باستخدام ثلاث مقاييس من مقاييس التطابق matching metric وهي : Absolute Distance

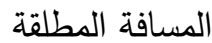

Euclidean Distance

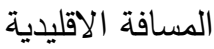

D1 Distance

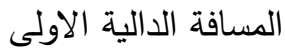

حيث قام البحث بمحاولة تحسين نسبة التمييز بالاعتمـاد على خواص الأبعاد الهندسية من خـلال زيـادة عدد الخواص التميزية وكانت النتائج التي تم الحصول عليها كما هو مبين في الجداول 2 و 3 حيث يبين الجدول 2 عدد الاثخاص وعدد الصور المستخدمة وعدد الصور المميزة بصورة خاطئة في كل طريقة من الطرق الثلاثة ، في حين يبين الجدول 3 عدد الاشخاص المميزين مع نسب التمييز التي تم الحصول عليها في كل طريقة من الطرق الثلاثة : الجدول (2)عدد الاشخاص وعدد الصور المستخدمة وعدد الصور المميزة بصورة خاطئة في كل طريقة من الطرق الثلاثة 


\begin{tabular}{|c|c|c|c|c|c|}
\hline | & & & \multicolumn{3}{|c|}{ همى عبد الرحمن حسّو و زينة نبيل الذطيب } \\
\hline 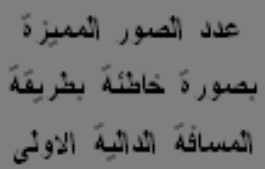 & 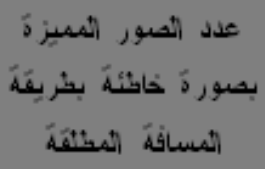 & 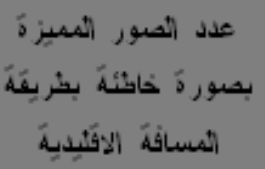 & 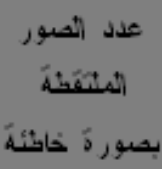 & عدل ألهور & الأشخاص \\
\hline 1 & 1 & 2 & 0 & 100 & 10 \\
\hline 6 & 4 & 12 & 3 & 200 & 20 \\
\hline 11 & 9 & 19 & 8 & 300 & 30 \\
\hline 15 & 14 & 28 & 12 & 400 & 40 \\
\hline 17 & 14 & 35 & 15 & 500 & 50 \\
\hline
\end{tabular}

الجدول (3) يبين عدد الاشخاص المميزين مع نسب التمييز

التي تم الحصول عليها في كل طريقة من الطرق الثلاثة

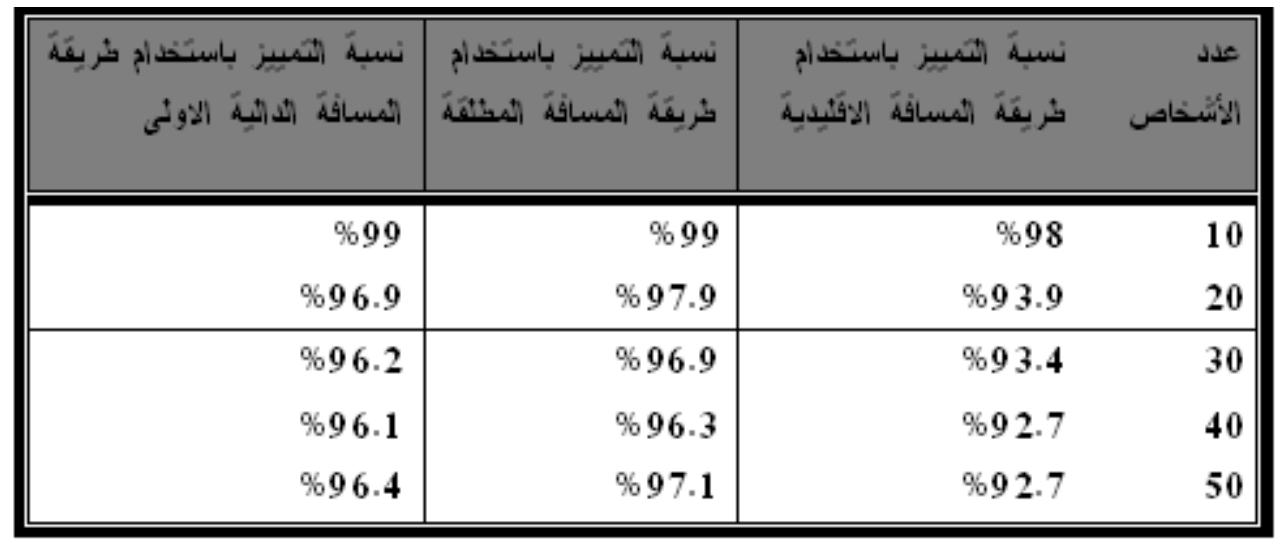

كما يبين الثكل (11) نسب التميز للطرق الثلاثة مع اختلاف حجم المجتمع المميز ، اذ نلاحظ انخفاض التاض

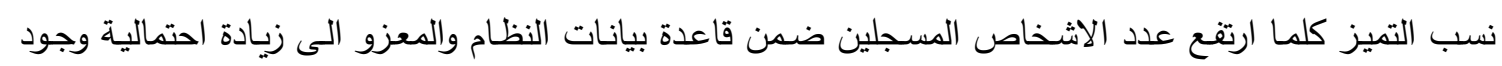
اشخاص متثابهين في حجم اليد وشكلها كلما ازداد عدد المسجلين ، كما ان الجدول (4) يبين مقارنة النتائج التي

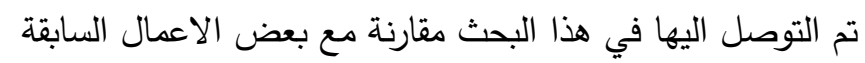

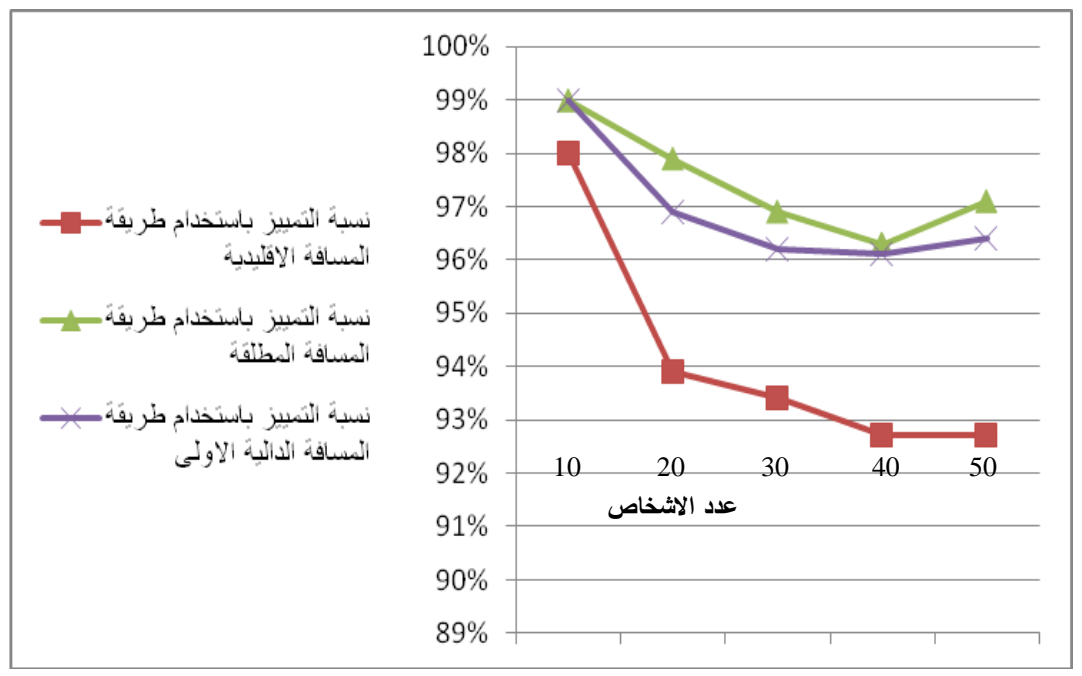


الثكل (11) نسب التميز للطرق الثلاثة مع اختلاف حجم المجتمع المميز

الجدول (4) يبين مقارنة النتائج التي تم التوصل اليها

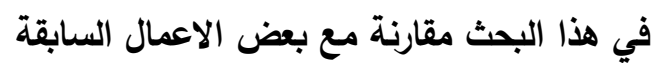

\begin{tabular}{|c|c|c|c|c|}
\hline نسبة التمييز & نوع التمييز & الأثخاص & حجم قاعدة & اسم البحث \\
\hline $\begin{array}{c}\mathrm{FAR}=0.1812 \% \\
\mathrm{FRR}=14.583 \% \\
\mathrm{EER}=4.62\end{array}$ & تأكد من هوية & 24 & 405 & $\begin{array}{c}\text { Using of Hand Geometry } \\
\text { in Biometric Security } \\
\text { Systems }\end{array}$ \\
\hline$R R=87.44 \%$ & تأكد من هوية & 13 & 78 & $\begin{array}{c}\text { Personal Authentication } \\
\text { Based On Hand Geometry } \\
\text { Verification }\end{array}$ \\
\hline$R R=85 \%$ & تأكد من هوية & 20 & 200 & $\begin{array}{c}\text { Access Control System } \\
\text { With Hand Geometry } \\
\text { Verification And } \\
\text { Smartcard } \\
\end{array}$ \\
\hline $\begin{array}{c}\mathrm{TA}=89 \% \\
\mathrm{FA}=2.2 \% \\
\text { HitRate }=96 \% \\
\text { FAR }=4.9 \%\end{array}$ & تأكد من هوية & اختبار 72 منهم & لمنها 3523استخدم 35 & $\begin{array}{l}\text { Peg-free Hand Geometry } \\
\text { Recognition Using } \\
\text { Hierachical Geometry and } \\
\text { Shape Matching }\end{array}$ \\
\hline 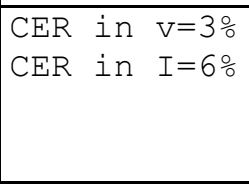 & تاكد من الهويـة & 96 & لم تذكر & $\begin{array}{c}\text { Personal Verification and } \\
\text { Identification Using Hand } \\
\text { Geometry }\end{array}$ \\
\hline $\mathrm{RR}=97.11 \%$ & تعرف على الثخص & 50 & 500 & 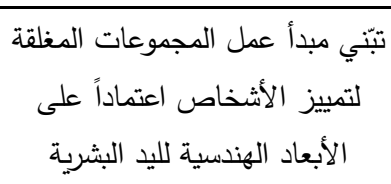 \\
\hline
\end{tabular}

المصادر

دسوقي فايزة احمد ،(2010) ، "القياسات الحيوية وامن المعلومات ", المؤتمر السادس لجمعية المكتبات

ستيف ويلان، بمساهمة من كل من موظفي الموسوعة الاستشارية لمساعدة الفقراء CGAP و شركة

Echange LLC ، "تكنولوجيا المقاييس الحيوية " ، سلسلة ابتكارات تكنولوجيا المعلومات التي تصدرها

$$
\text { الموسوعة الاستثارية لمساعدة الفقراء }
$$

\section{www.cgap.org/gm/document-1.9.2760/IT_bio_ar.pdf.}

[3]. Avinash Sharma, Nishant Shobhit and Anoop Namboodiri,(2008), "Projected Texture for Hand Geometry based Authentication", Center for Visual Information Technology, International Institute of Information Technology, Hyderabad, INDIA . 
[4]. Boukhonine Serguei, Krotov Vlad and Rupert Barry, (2005), "Future Security Approaches And Biometrics", Communications of the Association for Information Systems, Volume 16, pp., (937- 966).

[5]. Faundez-Zanuy, M.,(2005), “ Biometric Verification of Humans by Means of Hand Geometry", Security technology, CCST '05. In Proceedings of the 39th annual 2005 International Carnahan Conference on 11-14 October 2005.1-7

[6]. Jain Anil K. and Prabhakar Salil, (2004), "An Introduction to Biometric Recognition",IEEE Transactions on Circuits and systems for Video Technology, VOL. 14, NO. 1, pp (4-20).

[7]. Jain Anil. K, Ross Arun, and Pankanti Sharath ,(1999), "A Prototype Hand Geometry-Based Verification System", Proc. of 2nd Int'l Conference on Audio and Videobased, Biometric Person Authentication (AVBPA), pp.,(166-171).

[8]. Nongluk Covavisaruch, Pipat Prateepamornkul,Puripant Ruchikachorn, and Piyanaat Taksaphan,(2005), "Personal Verification and Identification Using Hand Geometry", Ecti Transactions On Computer And Information Technology VOL.1, NO.2 , pp., (134- 140).

[9]. Reillo Rand Sanchez and Marcos Ana Gonzalez,(2000), "Access Control System With Hand Geometry Verification And Smartcard",IEEE AES System Magazine , pp., (45- 48).

[10] Ríha Zdenek and Matyáš Václav, (2000), "Biometric Authentication Systems", FI MU Report Series, Masaryk University

[11]. Roberts Chris, (2006), "Biometric Technologies - Palm and Hand", http://www.ccip.govt.nz/newsroom/information-notes/2006/biometricstechnologies-palmhand.pdf

[12]. Ross Gary ,(2001), "Biometrics: A Self-Service Viewpoint” Biometrics and the ATM, Advanced Technology Group, NCR FSD, Dundee .

[13]. Roy Vandana and Jawahar C. V., (2005), "Feature Selection for HandGeometry based Person Authentication", Centre for Visual Information Technology, International Institute of Information Technology, Gachibowli, Hyderabad, India.

[14]. Statement of Keith A Rhodes, (2003), "INFORMATION SECURITY; Challenges in Using Biometrics", Chief Technologist, Applied Research and Methods,GAO-03.

[15]. VARCHOL Peter, LEVICKÝ Dušan, (2007), "Using of Hand Geometry in Biometric Security Systems", Radioengineering, VOL. 16, NO. 4, pp(82-87).

[16]. Wong Alexandra L.N , Shi Pengcheng (2002), "Peg-free Hand Geometry Recognition Using Hierarchical Geometry and Shape Matching”, In: IAPR workshop on machine vision Applications, Nara, Japan, pp.,(281-284) . 
[17]. Woodward John D., Jr., Horn Christopher, Gatune Julius, and Thomas Aryn, (2003), "Biometrics; A Look at Facial Recognition", RAND Public Safety and Justice.

[18]. Zhiu Chua Boon, (2010), "Personal Authentication Based On Hand Geometry Verification", Project Of Bachelor Of Electrical Engineering (Mechatronics), Faculty Of Electrical Engineering, University Teknologi Malaysia. 